International Journal of Applied Mathematics

Volume 26 No. $2 \quad 2013,159-175$

ISSN: 1311-1728 (printed version); ISSN: 1314-8060 (on-line version)

doi: http://dx.doi.org/10.12732/ijam.v26i2.3

\title{
STABILITY OF MIXED TYPE ADDITIVE QUADRATIC FUNCTIONAL EQUATION IN RANDOM NORMED SPACE
}

S. Murthy ${ }^{1}$, M. Arunkumar ${ }^{2 \S}$, G. Ganapathy ${ }^{3}$, P. Rajarethinam ${ }^{4}$

${ }^{1}$ Department of Mathematics

Government Arts College for Men

Krishnagiri, 635 001, Tamil Nadu, INDIA

${ }^{2}$ Department of Mathematics

Government Arts College

Tiruvannamalai, 606 603, Tamil Nadu, INDIA

${ }^{3}$ Department of Mathematics

R.M.D. Engineering College

Kavaraipettai, 601 206, Tamil Nadu, INDIA

${ }^{4}$ Department of Mathematics

Vysya College

Salem, 103, Tamil Nadu, INDIA

Abstract: In this paper, the authors obtain the general solution in vector space and generalized Ulam-Hyers stability of mixed type additive quadratic functional equation

$$
f(2 x \pm y \pm z)=2 f(-x \mp y \mp z)-2 f(\mp y \mp z)+f( \pm y \pm z)+3 f(x)-f(-x)
$$

in random normed space.

AMS Subject Classification: 39B52, 39B82

Key Words: additive functional equation, quadratic functional equation, AQ-functional equation, Generalized Ulam-Hyers-Rassias stability, RN-space

Received: February 11, $2013 \quad$ (c) 2013 Academic Publications

${ }^{\S}$ Correspondence author 


\section{Introduction}

A basic question in the theory of functional equations is as follows: when is it true that a function, which approximately satisfies a functional equation, must be close to an exact solution of the equation?

If the problem accepts a unique solution, we say the equation is stable.

The first stability problem concerning group homomorphisms was raised by S.M. Ulam [26] in 1940 and affirmatively solved by D.H. Hyers [12]. The result of Hyers was generalized by T. Aoki [2] for approximate additive mappings and by Th.M. Rassias [21] for approximate linear mappings by allowing the Cauchy difference operator to be controlled by $\varepsilon\left\{\|x\|^{p}+\|y\|^{p}\right\}$. In 1994, a generalization of Rassias theorem was obtained by P. Gavruta [9] who replaced $\varepsilon\left\{\|x\|^{p}+\|y\|^{p}\right\}$ by a general control function $\phi(x, y)$.

In addition, J.M. Rassias [20, 23] generalized the Hyers stability result by introducing two weaker conditions controlled by a product of different powers of norms and a mixed product sum of powers of norms, respectively. Recently, several further interesting discussions, modifications, extensions, and generalizations of the original problem of Ulam have been proposed, see $[1,13,18,22]$ and the references therein.

K.W. Jun and H.M. Kim [14] introduced the following generalized quadratic and additive type functional equation

$$
f\left(\sum_{i=1}^{n} x_{i}\right)+(n-2) \sum_{i=1}^{n} f\left(x_{i}\right)=\sum_{1 \leq i<j \leq n} f\left(x_{i}+x_{j}\right)
$$

in the class of function between real vector spaces. For $n=3$, Pl. Kannappan proved that a function $f$ satisfies the functional equation (1.1) if and only if there exists a symmetric bi-additive function $A$ and additive function $B$ such that $f(x)=B(x, x)+A(x)$ for all $x$ (see [17]). The Hyers-Ulam stability for the equation (1.1) when $n=3$ was proved by S.M. Jung [15]. The Hyers-UlamRassias stability for the equation (1.1) when $n=4$ was also investigated by I.S. Chang et al., [7]. A. Najati and M.B. Moghimi [19] investigated the generalized Hyers-Ulam-Rassias stability for the quadratic and additive type functional equation of the form

$$
f(2 x+y)+f(2 x-y)=2 f(x+y)+2 f(x-y)+2 f(2 x)-4 f(x) .
$$

The general solution and generalized Ulam-Hyers stability of a mixed type Additive Quadratic(AQ)-functional equation

$$
g(x+y)+g(x-y)=2 g(x)+g(y)+g(-y),
$$


was investigated by M. Arunkumar and J.M. Rassias [6].

Definition 1.1. Let $X$ and $Y$ be real vector spaces and let $f: X \rightarrow Y$, then:

(1) $f$ is said to be Arun-Additive Functional Equation [3] if $f$ satisfies the functional equation

$$
f(2 x \pm y \pm z)=f(x \pm y)+f(x \pm z)
$$

for all $x, y, z \in X$.

(2) $f$ is said to be Arun-Quadratic Functional Equation [4] if $f$ satisfies the functional equation

$$
f(2 x \pm y \pm z)+2 f(y)+2 f(z)=2 f(x \pm y)+2 f(x \pm z)+f(y+z)
$$

for all $x, y, z \in X$.

(3) $f$ is said to be Arun-Cubic Functional Equation [5] if $f$ satisfies the functional equation

$$
\begin{array}{r}
f(2 x \pm y \pm z)+f( \pm y \pm z)+2 f( \pm y)+2 f( \pm z)=2 f(x \pm y \pm z) \\
+f(x \pm y)+f(x \pm z)+f(-x \pm y)+f(-x \pm z)+6 f(x)
\end{array}
$$

for all $x, y, z \in X$.

(4) $f$ is said to be Arun-Additive Quadratic (AQ) Functional Equation, if $f$ satisfies the functional equation

$$
\begin{aligned}
& f(2 x \pm y \pm z) \\
& \quad=2 f(-x \mp y \mp z)-2 f(\mp y \mp z)+f( \pm y \pm z)+3 f(x)-f(-x)
\end{aligned}
$$

for all $x, y, z \in X$.

In this paper, the authors have established the general solution in vector space and generalized Ulam-Hyers stability of AQ-functional equation (1.7) in random normed spaces. It is easy to see that the mapping $f(x)=a x+b x^{2}$ is a solution of the functional equation (1.7).

In Section 2, we investigate the general solution of functional equation (1.7) when $f$ is a mapping between vector spaces.

In Section 3, we present preliminaries, notations and conventions of the theory of random normed spaces and, in Section 4, we establish the stability of the functional equation (1.7) in $\mathrm{RN}$-spaces. 


\section{General Solution of the Functional Equation (1.7)}

In this section, the general solution of the functional equation (1.7) is given.

Theorem 2.1. Let $X$ and $Y$ be real vector spaces. The odd mapping $f: X \rightarrow Y$ satisfies the functional equation

$$
f(x+y)=f(x)+f(y)
$$

for all $x, y \in X$ if and only if $f: X \rightarrow Y$ satisfies the functional equation

$$
f(2 x \pm y \pm z)=2 f(-x \mp y \mp z)-2 f(\mp y \mp z)-f( \pm y \pm z)+3 f(x)-f(-x)
$$

for all $x, y, z \in X$ with $f(0)=0$.

Proof. Let $f: X \rightarrow Y$ satisfy the functional equation (2.1). Setting $(x, y)$ by $(0,0)$ in $(2.1)$, we obtain $f(0)=0$. Replacing $y$ by $x$ and $y$ by $2 x$ in (2.1), we obtain

$$
f(2 x)=2 f(x) \quad \text { and } \quad f(3 x)=3 f(x)
$$

for all $x \in X$. In general for any positive integer $a$, we have

$$
f(a x)=a f(x)
$$

for all $x \in X$. Replacing $(x, y)$ by $(x, x \pm y \pm z)$ in (2.1), we get

$$
f(2 x \pm y \pm z)=f(x) \pm f(x+y+z)
$$

for all $x, y, z \in X$. Using (2.1) in (2.5), we obtain

$$
f(2 x \pm y \pm z)=2 f(x) \pm f(y+z)
$$

for all $x, y, z \in X$. Adding $2 f(x)$ and $2 f(\mp y \mp z)$ on both sides of (2.6), we arrive at

$$
\begin{aligned}
f(2 x \pm y \pm z)+2 f(x)+2 f(\mp y \pm z) & \\
& =2 f(x) \pm f(y+z)+2 f(x)+2 f(\mp y \mp z)
\end{aligned}
$$

for all $x, y, z \in X$. Equation (2.7) can be rewritten as

$$
\begin{aligned}
& f(2 x \pm y \pm z) \\
& \quad=2 f(x) \pm f(y+z)+2 f(x)+2 f(\mp y+\mp z)-2 f(x)-2 f(\mp y+\mp z)
\end{aligned}
$$


for all $x, y, z \in X$. Using (2.1) in (2.8), we obtain

$$
f(2 x \pm y \pm z)=2 f(-x \mp y \mp z)-2 f(\mp y \mp z)+f( \pm y \pm z)+3 f(x)-f(-x)
$$

for all $x, y, z \in X$.

Conversely, $f: X \rightarrow Y$ satisfies the functional equation (2.2) with $f(0)=0$. Setting $z$ by 0 and using oddness of $f$ in (2.2), we get

$$
f(2 x \pm y)=2 f(-x \mp y)+4 f(x) \mp 3 f(y)
$$

for all $x, y \in X$. Replacing $(x, y)$ by $(x, 0)$ and $(x, \mp x)$ respectively in $(2.10)$, we obtain

$$
f(2 x)=2 f(x) \text { and } \quad f(3 x)=3 f(x)
$$

for all $x \in X$. In general for any positive integer $b$, we have

$$
f(b x)=b f(x)
$$

for all $x \in X$. Substituting $(x, y)$ by $(x, \mp x \pm y)$ and remodifying (2.10), we get

$$
f(x+y)+3 f(x-y)=-2 f(y)+4 f(x)
$$

for all $x, y \in X$. Interchanging $x$ and $y$ in (2.13) and using oddness of $f$, we obtain

$$
f(x+y)-3 f(x-y)=-2 f(x)+4 f(y)
$$

for all $x, y \in X$. Adding (2.13) and (2.14), we arrive at (2.1), for all $x, y \in$ $X$.

Theorem 2.2. Let $X$ and $Y$ be real vector spaces. The even mapping $f: X \rightarrow Y$ satisfies the functional equation

$$
f(x+y)+f(x-y)=2 f(x)+2 f(y)
$$

for all $x, y \in X$ if and only if $f: X \rightarrow Y$ satisfies the functional equation

$$
f(2 x \pm y \pm z)=2 f(-x \mp y \mp z)-2 f(\mp y \mp z)-f( \pm y \pm z)+3 f(x)-f(-x)
$$

for all $x, y, z \in X$ with $f(0)=0$.

Proof. Let $f: X \rightarrow Y$ satisfy the functional equation (2.15). Setting $(x, y)$ by $(0,0)$ in $(2.15)$, we obtain $f(0)=0$. Replacing $y$ by $x$ and $2 x$ in $(2.15)$, we arrive

$$
f(2 x)=4 f(x) \text { and } \quad f(3 x)=9 f(x)
$$


for all $x \in X$. In general for any positive integer $a$, we have

$$
f(a x)=a^{2} f(x)
$$

for all $x \in X$. Replacing $(x, y)$ by $(x, x \pm y \pm z)$ in (2.15), we get

$$
f(2 x \pm y \pm z)=2 f(x \pm y \pm z)+2 f(x)-f( \pm y \pm z)
$$

for all $x, y, z \in X$. Adding $2 f(x)$ and $f( \pm y \pm z)$ on both sides of (2.19), we get

$$
\begin{aligned}
f(2 x \pm y & \pm z)+f(x)+f( \pm y+ \pm z) \\
& =2 f(x \pm y \pm z)+2 f(x)-f( \pm y \pm z)+f(x)+f( \pm y+ \pm z)
\end{aligned}
$$

for all $x, y, z \in X$. Using evenness of $f$ in (2.21), we arrive

$$
f(2 x \pm y \pm z)=2 f(-x \mp y \mp z)-2 f( \pm y \pm z)-f( \pm y+ \pm z)+3 f(x)-f(-x)
$$

for all $x, y, z \in X$.

Conversely, $f: X \rightarrow Y$ satisfies the functional equation (2.16) with $f(0)=$ 0 . Replacing $z$ by 0 in $(2.16)$, we obtain

$$
f(2 x \pm y)=2 f(x \pm y)+2 f(x)-f(y)
$$

for all $x, y \in X$. Replacing $y$ by 0 and $\pm x$ in (2.15), we get

$$
f(2 x)=4 f(x) \quad \text { and } \quad f(3 x)=9 f(x)
$$

for all $x \in X$. In general for any positive integer $b$, we have

$$
f(b x)=b^{2} f(x)
$$

for all $x \in X$. Substituting $(x, y)$ by $(x, \mp x \pm y)$ in (2.23) and using evenness of $f$, we arrive (2.15) for all $x, y \in X$.

\section{Preliminaries}

In the sequel, we adopt the usual terminology, notations and conventions of the theory of random normed spaces as in [8, 24, 25].

Throughout this paper, $\Delta^{+}$is the space of distribution functions, that is, the space of all mappings $F: R \cup\{-\infty, \infty\} \rightarrow[0,1]$, such that $F$ is leftcontinuous and nondecreasing on $R, F(0)=0$ and $F(+\infty)=1$. $D^{+}$is a subset of $\Delta^{+}$ 
consisting of all functions $F \in \Delta^{+}$for which $l^{-} F(+\infty)=1$, where $l^{-} f(x)$ denotes the left limit of the function $f$ at the point $x$, that is, $l^{-} f(x)=\lim _{t \rightarrow x^{-}} f(t)$. The space $\Delta^{+}$is partially ordered by the usual pointwise ordering of functions, that is, $F \leq G$ if and only if $F(t) \leq G(t)$ for all $t \in \mathbb{R}$. The maximal element for $\Delta^{+}$in this order is the distribution function $\epsilon_{0}$ given by

$$
\epsilon_{0}(t)= \begin{cases}0, & \text { if } t \leq 0 \\ 1, & \text { if } t \geq 0\end{cases}
$$

Definition 3.1. (see [24]) A mapping $T:[0,1] \times[0,1] \rightarrow[0,1]$ is called a continuous triangular norm (briefly, a continuous $t-$ norm) if $T$ satisfies the following conditions:

(a) $T$ is commutative and associative;

(b) $T$ is continuous;

(c) $T(a, 1)=a$ for all $a \in[0,1]$;

(d) $T(a, b) \leq T(c, d)$ whenever $a \leq c$ and $b \leq d$ for all $a, b, c, d \in[0,1]$.

Typical examples of continuous $t$-norms are $T_{P}(a, b)=a b, T_{M}(a, b)=$ $\min (a, b)$ and $T_{L}(a, b)=\max (a+b-1,0)$ (the Lukasiewicz $t$-norm). Recall (see $[10,11])$ that if $T$ is a $t$-norm and $x_{n}$ is a given sequence of numbers in $[0,1]$, then $T_{i=1}^{n} x_{n+i}$ is defined recurrently by

$$
T_{i=1}^{1} x_{i}=x_{1} \text { and } T_{i=1}^{n} x_{i}=T\left(T_{i=1}^{n-1} x_{i}, x_{n}\right) \text { for } n \geq 2 .
$$

$T_{i=n}^{\infty} x_{i}$ is defined as $T_{i=1}^{\infty} x_{n+i}$. It is known [11] that, for the Lukasiewicz $t-$ norm, the following implication holds:

$$
\lim _{n \rightarrow \infty}\left(T_{L}\right)_{i=1}^{\infty} x_{n+i}=1 \Longleftrightarrow \sum_{n=1}^{\infty}\left(1-x_{n}\right)<\infty
$$

Definition 3.2. (see [25]) A random normed space (briefly, RN-space) is a triple $(X, \mu, T)$, where $X$ is a vector space, $T$ is a continuous $t$-norm and $\mu$ is a mapping from $X$ into $D^{+}$satisfying the following conditions:

(RN1) $\mu_{x}(t)=\varepsilon_{0}(t)$ for all $t>0$ if and only if $x=0$;

(RN2) $\mu_{\alpha x}(t)=\mu_{x}(t /|\alpha|)$ for all $x \in X$, and $\alpha \in \mathbb{R}$ with $\alpha \neq 0$; 
(RN3) $\mu_{x+y}(t+s) \geq T\left(\mu_{x}(t), \mu_{y}(s)\right)$ for all $x, y \in X$ and $t, s \geq 0$.

Example 3.3. Every normed spaces $(X,\|\cdot\|)$ defines a random normed space $\left(X, \mu, T_{M}\right)$, where

$$
\mu_{x}(t)=\frac{t}{t+\|x\|}
$$

and $T_{M}$ is the minimum $t$-norm. This space is called the induced random normed space.

Definition 3.4. Let $(X, \mu, T)$ be a $\mathrm{RN}$-space.

(1) A sequence $\left\{x_{n}\right\}$ in $X$ is said to be convergent to a point $x \in X$ if, for any $\varepsilon>0$ and $\lambda>0$, there exists a positive integer $N$ such that $\mu_{x_{n}-x}(\varepsilon)>1-\lambda$ for all $n \geq N$.

(2) A sequence $\left\{x_{n}\right\}$ in $X$ is called a Cauchy sequence if, for any $\varepsilon>0$ and $\lambda>0$, there exists a positive integer $N$ such that $\mu_{x_{n}-x_{m}}(\varepsilon)>1-\lambda$ for all $n \geq m \geq N$.

(3) A RN-space $(X, \mu, T)$ is said to be complete if every Cauchy sequence in $X$ is convergent to a point in $X$.

Theorem 3.5. (see [25]) If $(X, \mu, T)$ is a $R N$-space and $\{x n\}$ is a sequence in $X$ such that $x_{n} \rightarrow x$, then $\lim _{n \rightarrow \infty} \mu_{x_{n}}(t)=\mu_{x}(t)$ almost everywhere.

\section{Stability Results in RN - Space}

In this section, the generalized Ulam-Hyers stability of the AQ-functional equation (1.7) in RN-space is provided. Let us consider $X$ to be a linear space and $(Y, \mu, T)$ to be a complete RN-space. Define a mapping $F: X \rightarrow Y$ by

$$
\begin{aligned}
F(x, y, z) & =f(2 x \pm y \pm z)-2 f(-x \mp y \mp z)+2 f(\mp y \mp z) \\
& +f( \pm y \pm z)+3 f(x)-f(-x)
\end{aligned}
$$

for all $x, y, z, \in X$. 
Theorem 4.1. Let $j= \pm 1$. Let $f_{a}: X \rightarrow Y$ be an odd mapping for which there exists a function $\eta: X^{3} \rightarrow D^{+}$with the condition

$$
\lim _{n \rightarrow \infty} T_{i=0}^{\infty}\left(\eta_{2^{i j} x, 2^{i j} y, 2^{i j} z}\left(2^{(i+1) j} t\right)\right)=1=\lim _{n \rightarrow \infty} \eta_{2^{n j} x, 2^{n j} y, 2^{n j} z}\left(2^{n j} t\right)
$$

such that the functional inequality with $f_{a}(0)=0$ such that

$$
\mu_{F(x, y, z)}(t) \geq \eta_{x, y, z}(t)
$$

for all $x, y, z \in X$ and all $t>0$. Then there exists a unique additive mapping $A: X \rightarrow Y$ satisfying the functional equation (1.7) and

$$
\mu_{A(x)-f_{a}(x)}(t) \geq T_{i=0}^{\infty}\left(\eta_{2^{i j} x, 0,0}\left(2^{(i+1) j} t\right)\right)
$$

for all $x \in X$ and all $t>0$. The mapping $A(x)$ is defined by

$$
\mu_{A(x)}(t)=\lim _{n \rightarrow \infty} \mu_{\frac{f_{a}\left(2^{n j} x\right)}{2^{n j}}}(t)
$$

for all $x \in X$ and all $t>0$.

Proof. Assume $j=1$. Setting $y=z=0$ and using oddness of $f$ in (4.1), we get

$$
\mu_{f_{a}(2 x)-2 f_{a}(x)}(t) \geq \eta_{x, 0,0}(t)
$$

for all $x \in X$ and all $t>0$. It follows from (4.5) and (RN2), we have

$$
\mu_{\frac{f_{a}(2 x)}{2}-f_{a}(x)}(t) \geq \eta_{x, 0,0}(2 t)
$$

for all $x \in X$ and all $t>0$. Replacing $x$ by $2^{n} x$ in (4.6), we arrive

$$
\mu_{\frac{f_{a}\left(2^{n+1} x\right)}{2^{n+1}}-\frac{f_{a}\left(2^{n} x\right)}{2^{n}}}(t) \geq \eta_{2^{n} x, 0,0}\left(2^{n+1} t\right)
$$

for all $x \in X$ and all $t>0$. It is easy to see that

$$
\frac{f_{a}\left(2^{n} x\right)}{2^{n}}-f_{a}(x)=\sum_{i=0}^{n-1} \frac{f_{a}\left(2^{i+1} x\right)}{2^{i+1}}-\frac{f_{a}\left(2^{i} x\right)}{2^{i}}
$$

for all $x \in X$. From equations (4.7) and (4.8), we have

$$
\begin{aligned}
\mu_{\frac{f_{a}\left(2^{n} x\right)}{2^{n}}-f_{a}(x)}(t) & =\mu_{\sum_{i=0}^{n-1} \frac{f_{a}\left(2^{i+1} x\right)}{2^{i+1}}-\frac{f_{a}\left(2^{i} x\right)}{2^{i}}}(t) \\
& \geq T_{i=0}^{n-1} \mu_{\frac{f_{a}\left(2^{i+1} x\right)}{2^{i+1}}-\frac{f_{a}\left(2^{i} x\right)}{2^{i}}}\left(\frac{t}{2^{i+1}}\right) \\
& \geq T_{i=0}^{n-1} \eta_{2^{i} x, 0,0}\left(2^{i+1} t\right)
\end{aligned}
$$


for all $x \in X$ and all $t>0$. In order to prove the convergence of the sequence $\left\{\frac{f_{a}\left(2^{n} x\right)}{2^{n}}\right\}$, we replace $x$ by $2^{m} x$ in (4.9), we arrive

$$
\begin{aligned}
\mu_{\frac{f_{a}\left(2^{n+m} x\right)}{2^{n+m}}-f_{a}(x)}(t) & \geq T_{i=0}^{n-1} \eta_{2^{i+m} x, 0,0}\left(2^{i+m+1} t\right) \\
& =T_{i=m}^{m+n-1} \eta_{2^{i} x, 0,0}\left(2^{i+1} t\right) \\
& \rightarrow 0 \text { as } m \rightarrow \infty
\end{aligned}
$$

for all $x \in X$ and all $t>0$. Thus $\left\{\frac{f_{a}\left(2^{n} x\right)}{2^{n}}\right\}$ is a Cauchy sequence. Since $Y$ is complete there exists a mapping $A: X \rightarrow Y$, we define

$$
\mu_{A(x)}(t)=\lim _{n \rightarrow \infty} \mu_{\frac{f_{a}\left(2^{n} x\right)}{2^{n}}}(t)
$$

for all $x \in X$ and all $t>0$. Letting $m=0$ and $n \rightarrow \infty$ in (4.10), we arrive (4.3) for all $x \in X$ and all $t>0$. Now, we have to show that $A$ satisfies (1.7), replacing $(x, y, z)$ by $\left(2^{n} x, 2^{n} y, 2^{n} z\right)$, we have

$$
\mu_{F\left(2^{n} x, 2^{n} y, 2^{n} z\right)}(t) \geq \eta_{2^{n} x, 2^{n} y, 2^{n} z}\left(2^{n} t\right)
$$

for all $x \in X$ and all $t>0$. Taking $n \rightarrow \infty$ both sides, we find that $A$ satisfies (1.7) for all $x, y, z \in X$. Therefore the mapping $A: X \rightarrow Y$ is additive.

Finally, to prove the uniqueness of the additive function $A$ subject to (4.4), let us assume that there exist a additive function $A^{\prime}$ which satisfies (4.3) and (4.4). Since $A\left(2^{n} x\right)=2^{n} A(x)$ and $A^{\prime}\left(2^{n} x\right)=2^{n} A^{\prime}(x)$ for all $x \in X$ and all $n \in \mathbb{N}$, it follows from (4.4) that

$$
\begin{aligned}
\mu_{A(x)-A^{\prime}(x)}(2 t) & =\mu_{A\left(2^{n} x\right)-A^{\prime}\left(2^{n} x\right)}\left(2^{n+1} t\right) \\
& =\mu_{A\left(2^{n} x\right)-f_{a}\left(2^{n} x\right)+f_{a}\left(2^{n} x\right)-A^{\prime}\left(2^{n} x\right)}\left(2^{n+1} t\right) \\
& \geq T\left(\mu_{A\left(2^{n} x\right)-f_{a}\left(2^{n} x\right)}\left(2^{n+1} t\right), \mu_{f_{a}\left(2^{n} x\right)-A^{\prime}\left(2^{n} x\right)}\left(2^{n+1} t\right)\right) \\
& =T\left(T_{i=0}^{\infty}\left(\eta_{2^{i} x, 0,0}\right)\left(2^{i+1} t\right), T_{i=0}^{\infty}\left(\eta_{2^{i} x, 0,0}\right)\left(2^{i+1} t\right)\right) \\
& \rightarrow 0 \text { as } n \rightarrow \infty
\end{aligned}
$$

for all $x \in X$ and all $t>0$. Hence $A$ is unique.

For $j=-1$, we can prove a similar stability result. This completes the proof of the theorem.

The following corollary is an immediate consequence of Theorem 4.1 concerning the stability of (1.7). 
Corollary 4.2. Let $\lambda$ and $r$ be nonnegative real numbers. Let an odd function $f_{a}: X \rightarrow Y$ satisfy the inequality

$$
\mu_{F(x, y, z)}(s) \geq \begin{cases}\eta_{\lambda}(s) & ; r<1 \text { or } r>1 \\ \eta_{\lambda\left(\left.\|x\|\right|^{r}+\|y\|^{r}+\| z||^{r}\right)}(s) & ; r<\frac{1}{3} \text { or } r>\frac{1}{3} \\ \eta_{\lambda\left[\left.\|x\|\right|^{r}|| y||^{r} \mid z z \|^{r}+\left(\left\|x||^{3 r}+\right\| y\left\|^{3 r}+\right\| z \|^{3 r}\right)\right]}(s) & \end{cases}
$$

for all $x, y, z \in X$ and all $s>0$. Then there exists a unique additive mapping $A: X \rightarrow Y$ satisfying the functional equation (1.7) and

$$
\mu_{A(x)-f_{a}(x)}(s) \geq\left\{\begin{array}{l}
\eta_{\lambda}(s) \\
\eta_{\frac{\lambda\|x\|^{r}}{\left|2-2^{r}\right|}}(s) \\
\frac{\eta_{\lambda \| x||^{3 r}}(s)}{\left|2-2^{3 r}\right|}
\end{array}\right.
$$

for all $x \in X$ and all $s>0$.

Theorem 4.3. Let $j= \pm 1$. Let $f: X \rightarrow Y$ be an even mapping for which there exists a function $\eta: X^{3} \rightarrow D^{+}$with the condition

$$
\lim _{n \rightarrow \infty} T_{i=0}^{\infty}\left(\eta_{2^{i j} x, 2^{i j} y, 2^{i j} z}\left(4^{(i+1) j} t\right)\right)=1=\lim _{n \rightarrow \infty} \eta_{2^{n j} x, 2^{n j} y, 2^{n j} z}\left(4^{n j} t\right)
$$

such that the functional inequality with $f_{q}(0)=0$ such that

$$
\mu_{F(x, y, z)}(t) \geq \eta_{x, y, z}(t)
$$

for all $x, y, z \in X$ and all $t>0$. Then there exists a unique quadratic mapping $Q: X \rightarrow Y$ satisfying the functional equation (1.7) and

$$
\mu_{Q(x)-f_{q}(x)}(t) \geq T_{i=0}^{\infty}\left(\eta_{2^{i j} x, 0,0}\left(4^{(i+1) j} t\right)\right)
$$

for all $x \in X$ and all $t>0$. The mapping $Q(x)$ is defined by

$$
\mu_{Q(x)}(t)=\lim _{n \rightarrow \infty} \mu_{\frac{f_{q}\left(2^{n j} x\right)}{4^{n j}}}(t)
$$

for all $x \in X$ and all $t>0$.

Proof. Assume $j=1$. Setting $y=z=0$ and using the evenness of $f$ in (4.14), we get

$$
\mu_{f_{q}(2 x)-4 f_{q}(x)}(t) \geq \eta_{x, 0,0}(t)
$$


for all $x \in X$ and all $t>0$. It follows from (4.18) and (RN2), we have

$$
\mu_{\frac{f_{q}(2 x)}{4}-f_{q}(x)}(t) \geq \eta_{x, 0,0}(4 t)
$$

for all $x \in X$ and all $t>0$. Replacing $x$ by $2^{n} x$ in (4.19), we arrive at

$$
\mu_{\frac{f q\left(2^{n+1} x\right)}{4^{n+1}}-\frac{f_{q}\left(2^{n} x\right)}{4^{n}}}(t) \geq \eta_{2^{n} x, 0,0}\left(4^{n+1} t\right)
$$

for all $x \in X$ and all $t>0$. It is easy to see that

$$
\frac{f_{q}\left(2^{n} x\right)}{4^{n}}-f_{q}(x)=\sum_{i=0}^{n-1} \frac{f_{q}\left(2^{i+1} x\right)}{4^{i+1}}-\frac{f_{q}\left(2^{i} x\right)}{4^{i}}
$$

for all $x \in X$. From equations (4.20) and (4.21), we have

$$
\begin{aligned}
\mu_{\frac{f_{q}\left(2^{n} x\right)}{4^{n}}-f_{q}(x)}(t) & =\mu_{\sum_{i=0}^{n-1} \frac{f_{q}\left(2^{i+1} x\right)}{4^{i+1}}-\frac{f_{q}\left(2^{i} x\right)}{4^{i}}}(t) \\
& \geq T_{i=0}^{n-1} \mu_{\frac{f_{q}\left(2^{i+1} x\right)}{4^{i+1}}-\frac{f_{q}\left(2^{i} x\right)}{4^{i}}}\left(\frac{t}{4^{i+1}}\right) \\
& \geq T_{i=0}^{n-1} \eta_{2^{i} x, 0,0}\left(4^{i+1} t\right)
\end{aligned}
$$

for all $x \in X$ and all $t>0$. In order to prove the convergence of the sequence $\left\{\frac{f_{q}\left(2^{n} x\right)}{4^{n}}\right\}$, we replace $x$ by $2^{m} x$ in $(4.22)$, we arrive at

$$
\begin{aligned}
\mu_{\frac{f_{q}\left(2^{n+m} x\right)}{4^{n+m}}-f_{q}(x)}(t) & \geq T_{i=0}^{n-1} \eta_{2^{i+m} x, 0,0}\left(4^{i+m+1} t\right) \\
& =T_{i=m}^{m+n-1} \eta_{2^{i} x, 0,0}\left(4^{i+1} t\right) \\
& \rightarrow 0 \text { as } m \rightarrow \infty
\end{aligned}
$$

for all $x \in X$ and all $t>0$. Thus $\left\{\frac{f_{q}\left(2^{n} x\right)}{4^{n}}\right\}$ is a Cauchy sequence. Since $Y$ is complete, there exists a mapping $Q: X \rightarrow Y$, we define

$$
\mu_{Q(x)}(t)=\lim _{n \rightarrow \infty} \mu_{\frac{f_{q}\left(2^{\left.n j_{x}\right)}\right.}{4^{n j}}}(t)
$$

for all $x \in X$ and all $t>0$. Letting $m=0$ and $n \rightarrow \infty$ in (4.23), we arrive (4.16) for all $x \in X$ and all $t>0$. In order to show that $Q$ satisfies (1.7) and it is unique the proof is similar to that of Theorem 4.1.

For $j=-1$, we can prove a similar stability result. This completes the proof of the theorem. 
The following corollary is an immediate consequence of Theorem 4.3 concerning the stability of (1.7).

Corollary 4.4. Let $\lambda$ and $r$ be nonnegative real numbers. Let an even function $f_{q}: X \rightarrow Y$ satisfies the inequality

$$
\mu_{F(x, y, z)}(s) \geq \begin{cases}\eta_{\lambda}(s) & ; r<1 \text { or } r>1 \\ \eta_{\lambda\left(\left\|x||^{r}+\right\| y\left\|^{r}+|| z\right\|^{r}\right)}(s) & ; r<\frac{1}{3} \text { or } r>\frac{1}{3} \\ \eta_{\lambda\left[\left.\left.\|x\|\right|^{r}|| y\left\|^{r}\right\| z\right|^{r}+\left(\|x\|^{3 r}+\|y\|^{3 r}+\|z\|^{3 r}\right)\right]}(s) & \end{cases}
$$

for all $x, y, z \in X$ and all $s>0$. Then there exists a unique quadratic mapping $Q: X \rightarrow Y$ satisfying the functional equation (1.7) and

$$
\mu_{Q(x)-f_{q}(x)}(s) \geq\left\{\begin{array}{l}
\frac{\eta_{\frac{4 \lambda}{3}}}{3}(s) \\
\eta_{\lambda \| x||^{r}}(s) \\
\frac{\eta_{\lambda \| 2^{r \mid}||^{3 r}}(s)}{\left|4-2^{3 r}\right|}
\end{array}\right.
$$

for all $x \in X$ and all $s>0$.

Theorem 4.5. Let $j= \pm 1$. Let $f: X \rightarrow Y$ be a mapping for which there exists a function $\eta: X^{3} \rightarrow D^{+}$satisfying the conditions (4.1) and (4.14) and the functional inequality with $f(0)=0$ such that

$$
\mu_{F(x, y, z)}(t) \geq \eta_{x, y, z}(t)
$$

for all $x, y, z \in X$ and all $t>0$. Then there exists a unique additive mapping $A: X \rightarrow Y$ and a unique quadratic mapping $Q: X \rightarrow Y$ satisfying the functional equation (1.7) and

$$
\begin{aligned}
& \mu_{A(x)-Q(x)-f(x)}(2 t) \\
& \geq T\left(T_{i=0}^{\infty}\left[T\left(\eta_{2^{i j} x, 0,0}\left(2^{(i+1) j} t\right)\right),\left(\eta_{-2^{i j} x, 0,0}\left(2^{(i+1) j} t\right)\right)\right]\right. \\
& \left.\quad T_{i=0}^{\infty}\left[T\left(\eta_{2^{i j} x, 0,0}\left(4^{(i+1) j} t\right)\right),\left(\eta_{-2^{i j} x, 0,0}\left(4^{(i+1) j} t\right)\right)\right]\right)
\end{aligned}
$$

for all $x \in X$ and all $t>0$. The mapping $A(x)$ and $Q(x)$ are respectively defined in (4.4) and (4.17) for all $x \in X$ and all $t>0$.

Proof. Let $f_{o}(x)=\frac{f_{a}(x)-f_{a}(-x)}{2}$ for all $x \in X$. Then $f_{o}(0)=0$ and $f_{o}(-x)=-f_{o}(x)$ for all $x \in X$. Hence

$$
\mu_{F_{o}(x, y, z)}(2 t) \geq T\left(\eta_{x, y, z}(t), \eta_{-x,-y,-z}(t)\right)
$$


for all $x, y, z \in X$ and all $t>0$. By Theorem 4.1, we have

$$
\mu_{A(x)-f_{o}(x)}(2 t) \geq T_{i=0}^{\infty}\left[T\left(\eta_{2^{i j} x, 0,0}\left(2^{(i+1) j} t\right)\right),\left(\eta_{-2^{i j} x, 0,0}\left(2^{(i+1) j} t\right)\right)\right]
$$

for all $x \in X$ and all $t>0$.

Also, let $f_{e}(x)=\frac{f_{q}(x)+f_{q}(-x)}{2}$ for all $x \in X$. Then $f_{e}(0)=0$ and $f_{e}(-x)=f_{e}(x)$ for all $x \in x$. Hence

$$
\mu_{F_{e}(x, y, z)}(2 t) \geq T\left(\eta_{x, y, z}(t), \eta_{-x,-y,-z}(t)\right)
$$

for all $x, y, z \in X$ and all $t>0$. By Theorem 4.3, we have

$$
\mu_{Q(x)-f_{e}(x)}(2 t) \geq T_{i=0}^{\infty}\left[T\left(\eta_{2^{i j} x, 0,0}\left(4^{(i+1) j} t\right)\right),\left(\eta_{-2^{i j} x, 0,0}\left(4^{(i+1) j} t\right)\right)\right]
$$

for all $x \in X$ and all $t>0$. Define

$$
f(x)=f_{e}(x)+f_{o}(x)
$$

for all $x \in x$. From (4.29), (4.31) and (4.32), we arrive

$$
\begin{aligned}
\mu_{A(x)}-Q(x)-f(x) & (2 t) \\
& =\mu_{A(x)-Q(x)-f_{o}(x)-f_{e}(x)}(2 t) \\
& \geq T\left(\mu_{A(x)-f_{o}(x)}(t), \mu_{Q(x)-f_{e}(x)}(t)\right) \\
& \geq T\left(T_{i=0}^{\infty}\left[T\left(\eta_{2^{i j} x, 0,0}\left(2^{(i+1) j} \frac{t}{2}\right)\right),\left(\eta_{-2^{i j} x, 0,0}\left(2^{(i+1) j} \frac{t}{2}\right)\right)\right]\right. \\
& \left.\quad T_{i=0}^{\infty}\left[T\left(\eta_{2^{i j} x, 0,0}\left(4^{(i+1) j} \frac{t}{2}\right)\right),\left(\eta_{-2^{i j} x, 0,0}\left(4^{(i+1) j} \frac{t}{2}\right)\right)\right]\right)
\end{aligned}
$$

for all $x \in X$ and all $t>0$. Hence the theorem is proved.

The following corollary is an immediate consequence of Corollaries 4.2 and 4.6 concerning the stability of (1.7).

Corollary 4.6. Let $\lambda$ and $r$ be nonnegative real numbers. Let a function $f: X \rightarrow Y$ satisfy the inequality

$$
\mu_{F(x, y, z)}(s) \geq \begin{cases}\eta_{\lambda}(s) & ; r<2 \text { or } r>2 \\ \eta_{\lambda\left(\left.\|x\|\right|^{r}+\|y\|^{r}+\|z\|^{r}\right)}(s) & ; r<\frac{1}{2} \text { or } r>\frac{1}{2} \\ \eta_{\lambda\left[\left\|\left.x\right|^{r}\right\| y\left\|^{r}\right\| z \|^{r}+\left(\|x\|^{3 r}+\|y\|^{3 r}+\|z\|^{3 r}\right)\right]}(s) & \end{cases}
$$


for all $x, y, z \in X$ and all $s>0$. Then there exist a unique additive mapping $A: X \rightarrow Y$ and a unique quadratic mapping $Q: X \rightarrow Y$ satisfying the functional equation (1.7) and

$$
\mu_{A(x)-Q(x)-f(x)}(2 s) \geq\left\{\begin{array}{l}
\eta_{\lambda+\frac{4 \lambda}{3}}(s) \\
\eta_{\lambda \|\left. x\right|^{r}\left(\frac{1}{\left|2-2^{r}\right|}+\frac{1}{\left|4-2^{r}\right|}\right)}(s) \\
\eta_{\lambda \| x||^{3 r}\left(\frac{1}{\left|2-2^{3 r}\right|}+\frac{1}{\left|4-2^{3 r}\right|}\right)}(s)
\end{array}\right.
$$

for all $x \in X$ and all $s>0$.

\section{References}

[1] J. Aczel and J. Dhombres, Functional Equations in Several Variables, Cambridge Univ. Press, 1989.

[2] T. Aoki, On the stability of the linear transformation in Banach spaces, J. Math. Soc. Japan, 2 (1950), 64-66.

[3] M. Arunkumar, Solution and stability of Arun-Additive functional, International Journal Mathematical Sciences and Engineering Applications, 4, No 3 (August 2010), 33-46.

[4] M. Arunkumar, S. Jayanthi, S. Hema Latha, Stability of quadratic derivations of Arun-quadratic functional equation, International Journal Mathematical Sciences and Engineering Applications, 5, No. 5 (Sept. 2011), 433-443.

[5] M. Arunkumar, C. Devi Shyamala Mary, Solution and generalized UlamHyers stability of a cubic functional equation, Proc. of the "Heber International Conference on Applications of mathematics and Statistics" (2012), 46-55.

[6] M. Arunkumar, J.M. Rassias, On the generalized Ulam-Hyers stability of an AQ-mixed type functional equation with counter examples, Far East Journal of Applied Mathematics (Submitted).

[7] I.S. Chang, E.H. Lee, H.M. Kim, On the Hyers-Ulam-Rassias stability of a quadratic functional equations, Math. Ineq. Appl., 6, No 1 (2003), 87-95. 
[8] S.S. Chang, Y.J. Cho, and S.M. Kang, Nonlinear Operator Theory in Probabilistic Metric Spaces, Nova Science Publishers, Huntington, NY - USA, 2001.

[9] P. Gavruta, A generalization of the Hyers-Ulam-Rassias stability of approximately additive mappings, J. Math. Anal. Appl., 184 (1994), 431-436.

[10] O. Hadzic and E. Pap, Fixed Point Theory in Probabilistic Metric Spaces, Vol. 536 of Ser. Mathematics and Its Applications, Kluwer Academic, Dordrecht, 2001.

[11] O. Hadzic, E. Pap and M. Budincevic, Countable extension of triangular norms and their applications to the fixed point theory in probabilistic metric spaces, Kybernetika, 38, No 3 (2002), 363382.

[12] D.H. Hyers, On the stability of the linear functional equation, Proc. Nat. Acad. Sci. USA, 27 (1941), 222-224.

[13] D.H. Hyers, G. Isac, Th.M. Rassias, Stability of Functional Equations in Several Variables, Birkhauser, Basel, 1998.

[14] K.W. Jun and H.M. Kim, On the stability of an n-dimensional quadratic and additive type functional equation, Math. Ineq. Appl., 9, No 1 (2006), 153-165.

[15] S.M. Jung, On the Hyers-Ulam stability of the functional equations that have the quadratic property, J. Math. Anal. Appl., 222 (1998), 126-137.

[16] S.M. Jung, Hyers-Ulam-Rassias Stability of Functional Equations in Mathematical Analysis, Hadronic Press, Palm Harbor, 2001.

[17] Pl. Kannappan, Quadratic functional equation inner product spaces, Results Math., 27, No 3-4, (1995), 368-372.

[18] Pl. Kannappan, Functional Equations and Inequalities with Applications, Springer Monographs in Mathematics, 2009.

[19] A. Najati, M.B. Moghimi, On the stability of a quadratic and additive functional equation, J. Math. Anal. Appl., 337 (2008), 399-415.

[20] J.M. Rassias, On approximately of approximately linear mappings by linear mappings, J. Funct. Anal. USA, 46 (1982), 126-130. 
[21] Th.M. Rassias, On the stability of the linear mapping in Banach spaces, Proc. Amer. Math. Soc., 72 (1978), 297-300.

[22] Th.M. Rassias, Functional Equations, Inequalities and Applications, Kluwer Acedamic Publishers, Dordrecht, Bostan London, 2003.

[23] K. Ravi, M. Arunkumar and J.M. Rassias, On the Ulam stability for the orthogonally general Euler-Lagrange type functional equation, International Journal of Mathematical Sciences, 3, No 8 (Autumn 2008), 36-47.

[24] B. Schweizer and A. Sklar, Probabilistic Metric Spaces, North-Holland Ser. in Probability and Applied Mathematics, North-Holland Publishing, New York, 1983.

[25] A.N. Sherstnev, On the notion of a random normed space, Doklady Akademii Nauk SSSR, 149 (1963) (Russian).

[26] S.M. Ulam, Problems in Modern Mathematics, Science Editions, Wiley, New York, 1964. 
